Gentner, D. \& Bowdle, B. F. (1994). The coherence imbalance hypothesis: A functional

approach to asymmetry in comparison. Proceedings of the Sixteenth Annual Conference of the Cognitive Science Society, 351-356. Hillsdale, NJ: Lawrence Erlbaum Associates.

\title{
The Coherence Imbalance Hypothesis: A Functional Approach to Asymmetry in Comparison
}

\author{
Dedre Gentner \\ Department of Psychology \\ Northwestern University \\ 2029 Sheridan Road \\ Evanston, Illinois 60208 \\ gentnerlnwu. edu
}

\author{
Brian F. Bowdle \\ Department of Psychology \\ Northwestern University \\ 2029 Sheridan Road \\ Evanston, Illinois 60208 \\ bowdlel nwu.edu
}

\begin{abstract}
Directional asymmetry is a well-documented phenomenon in research on similarity, metaphor, and analogy. In this paper, we present an account of this phenomenon based on structural alignment. We propose that a major source of asymmetry is coherence imbalance: that is, a difference in the degree of systematicity of the relational structures being compared. These claims are tested in three experiments which examine the relationship between asymmetry, informativity, and conceptual coherence. The results support the hypothesis that coherence imbalance is a key factor in directional comparison processes. Further, by incorporating the insights offered by structural alignment, coherence imbalance advances a more functional account of asymmetry.
\end{abstract}

Comparison is a fundamental process in cognitive theory, and is crucial to such aspects of mental operation as categorization, decision making, problem solving, and transfer of learning. Intuitively, comparisons might appear to be symmetric: $X$ is similar $10 Y$ implies that $Y$ is similar to $X$. Empirically, however, there is often a preference for one direction of comparison over the other. For example, Tversky (1977) found that subjects preferred the comparison North Korea is similar to Red China over the comparison Red China is similar to North Korea, and rated the similarity of North Korea to Red China as higher than that of Red China to North Korea. Asymmetries have been found not only in literal similarity, but in other forms of comparison, including metaphor and analogy. We begin by reviewing two influential models that have been proposed to explain such asymmetries.

According to Tversky's (1977) feature contrast model, the similarity of two entities increases as a function of their common (shared) features, and decreases as a function of their distinctive (non-shared) features. Asymmetries in literal similarity are accounted for in terms of the focusing hypothesis, which states that the target of a directional comparison is the focus of attention. ${ }^{1}$. Therefore, the distinctive features of the target are weighted more heavily than those of the base. This predicts that there is a general preference for placing the referent possessing the larger or

\footnotetext{
In directional comparisons of the form $A$ is like $B$, the aterm is referred to as the target and the b-term as the base.
}

more salient set of distinctive features in the base position of a comparison, as this ordering maximizes similarity.

To account for asymmetries in non-literal comparisons, Ortony (1979) proposed an extension of Tversky's model in his salience imbalance theory of metaphor. Unlike the feature contrast model, Ortony's model allows for the salience of the common features to vary across the referents of a comparison. On this account, metaphoricity arises from salience imbalance. Directional asymmetry in metaphor results from a preference for having the salience of matching features be higher in the base of a comparison than in the target. Thus, whereas the feature contrast model derives asymmetries from the distinctive feature sets, the salience imbalance model derives them from the common features.

Empirical tests of the salience imbalance model have yielded mixed results (Gentner \& Clement, 1988; Tourangeau \& Rips, 1991). More importantly, neither of the above models provides an account of how comparisons convey new information about their targets (Glucksberg \& Keysar, 1990). This issue is far from trivial, as in general use comparison statements are meant to be informative, often by suggesting inferences. In Tversky's model, the most obvious candidate for target inferences is the set of distinctive features of the base. However, no mechanism is provided for selecting any particular predication from the potentially large set of non-matching information. Ortony's model allows for predicate promotions, in which the salience of a shared attribute is raised in the target term due to its emphasis in the base. However, such promotions do not constitute truly novel information, and again there remains the problem of selection among potential predications.

We suggest that the problem of inference selection can best be approached by conceiving of the mental representations involved in comparisons as coherent relational structures (e.g., Keil, 1989; Murphy \& Medin, 1985) rather than as sets of isolated features. Further, a growing body of evidence suggests that the process of comparison is best characterized as a structural alignment of such relational systems (e.g., Markman \& Gentner, 1990, 1993; Medin, Goldstone, \& Gentner, 1993). This approach provides a mechanism for inference generation in similarity and analogy (Falkenhainer, Forbus, \& Gentner, 1989), and we propose that it is a significant factor in asymmetries. 


\section{The Coherence Imbalance Hypothesis}

According to the structural alignment view, similarity and other forms of comparison are processed by aligning and mapping structures, much as in structure-mapping in analogy (Gentner, 1983; 1989). Such comparisons tend to convey that a system of relations holding among the objects of the base domain also holds among the objects of the target domain: that is, similarity is determined not only on the basis of the intrinsic similarity of the elements comprising the base and target, but also by the extent to which these elements have corresponding roles in a common relational structure. This process of alignment is aimed at creating the maximal structurally consistent match between the two domains. Structural consistency involves one-toone mapping (i.e., an element in one representation can map onto no more than one element in the other); and parallel connectivity (i.e., for any pair of predicates that correspond, the arguments of these predicates must also correspond). A second aspect of the alignment process is the systematicity principle, which states that people prefer mappings that form coherent, interconnected structures, in which higherorder relations constrain lower-order relations.

According to structure-mapping theory, once a structurally consistent match between the base and target domains has been realized, further predicates from the base domain that are connected to the common system (but are not initially present in the target domain) can be mapped to the target as candidate inferences. Clement and Gentner (1991) have demonstrated that such inference selection can be predicted on the basis of connectivity. Subjects were presented with pairs of analogous passages, the base of which contained two facts that could readily map to the target. However, only one of these facts was embedded in a matching causal structure. When asked to make predictions about the target passages based on the analogies, subjects imported those facts that were linked to the common connected system, neglecting the equally available isolated facts. Thus, systematicity provides a basis for solving the problem of selection of candidate inferences.

As the above research implies, the process of drawing candidate inferences is essentially one of structural completion (Gentner \& Markman, 1993). Thus, if a comparison is to provide target predications, the base representation should possess more coherent structure - e.g., causal or explanatory connections - than the target representation. We propose that this is a major source of directionality in comparisons. When two concepts are placed in structural alignment, to the extent that the common relational system is more highly structured in one of the referents, the preferred direction of comparison will favor the more systematic or coherent referent as the base. In other words, asymmetries in comparison result from a coherence imbalance between the items being compared. This position provides a functional explanation of asymmetry: directional preferences reflect differences in the degree of informativity exhibited by each direction of comparison. To test the coherence imbalance hypothesis, we presented subjects with pairs of stories that varied in coherence, and asked them to select the direction of comparison displaying the greatest degree of similarity. Our prediction is that the more coherent story should be selected as the comparison base.

\section{Experiment 1}

For the experiments presented here, we constructed pairs of similar stories with varying degrees of coherence imbalance. To achieve some generality, the manner of creating coherence imbalance was varied. We designed sets of brief (one paragraph) stories, each containing a causally coherent standard story and three less coherent variants derived from it. Deletion variants (DVs) were created by eliminating a primary causal pivot in the standard story and substituting an innocuous, non-causal statement. Replacement variants (RVs) were created by eliminating the causal pivot and replacing it with another causal statement that was fairly incongruous with the story. Finally, shuffled variants (SVs) were created by rearranging the order of propositions in the standard story (while maintaining syntactic cohesion). Aside from differences in names and surface descriptions, the characters and locations contained in the variants corresponded closely to those of the standard stories. Further, the basic actions were kept the same, except for the modifications noted above. Thus, each of the variants was alignable with the standard. A sample story set is provided in Table 1.

Each story pair consisted of a standard and one of its three variants. The key prediction is that subjects should prefer the direction (less coherent) variant is similar to (more coherent) standard over the reverse. Further, we expected the degree of directional asymmetry to vary with the degree of coherence imbalance. Given the nature of the variant manipulations, we expected DV pairs to possess the lowest degree of coherence imbalance, and SV pairs the highest, with RV pairs somewhere in between. These intuitions were confirmed in a stimulus pretest, in which subjects were presented with story pairs and asked to rate the coherence of each story in each pair on a scale of 1 (low coherence) to 7 (high coherence). The coherence imbalance for a given pair was operationalized as the difference in coherence ratings between the standard and variant. Significantly lower coherence imbalance ratings were obtained for the DV pairs $(m=1.99)$ than for the $R V$ pairs $(m=2.70), t(17)=-2.90$, $p<.01$, and for the RV pairs than for the SV pairs $(m=4.19)$, $t(17)=6.24, p<.001$.

The coherence imbalance hypothesis predicts a directional similarity preference favoring the more coherent story as the base for all story comparisons. Further, since greater degrees of coherence imbalance should lead to greater asymmetries, the directionality should be weakest for DV pairs, and strongest for SV pairs.

\section{Method}

Subjects. Forty-nine Northwestern University undergraduates participated in partial fulfillment of a course requirement.

Materials. The stimuli were pairs of stories drawn from 18 basic story sets. Each pair consisted of a standard and one of the three variants described above (deletion, replacement, or 


\section{Standard}

Peter was the most faithful and devoted of all the younger monks in the monastery. He was as skilled at illuminating texts as he was at tending the gardens. His simple passion for God was admired by all. One day at dinner, he spilled his food onto the floor. Peter was dismayed. as he viewed the wasting of food as a sin. He went to his cubical and began to fast in silence. His elders became worried, but they decided to say nothing. When a week had passed, Peter felt that he had repented, and resumed his life as usual.

\section{Deletion Variant}

Luke was the most faithful and devoted of all the younger monks in the monastery. He was as skilled at chanting hymns as he was at copying texts. His simple passion for God was admired by all. His elders were particularly impressed by him, and expected that Luke would become a prominent figure. One day at dinner, he spilled his food onto the floor. He went to his cubical and began to fast in silence. His elders became worried, but they decided to say nothing. When a week had passed, Luke felt that he had repented, and resumed his life as usual.

\section{Replacement Variant}

Luke was the most faithful and devoted of all the younger monks in the monastery. He was as skilled at chanting hymns as he was at copying texts. His simple passion for God was admired by all. One day at dinner, he spilled his food onto the floor. Luke was dismayed. as the bean porridge they were serving that night was his favorite. He went to his cubical and began to fast in silence. His elders became worried, but they decided to say nothing. When a week had passed, Luke felt that he had repented, and resumed his life as usual.

\section{Shuffled Variant}

Luke was the most faithful and devoted of all the younger monks in the monastery. He was as skilled at chanting hymns as he was at copying texts. His simple passion for God was admired by all. Luke was dismayed. One day at dinner, he began to fast in silence, as he viewed the wasting of food as a sin. When a week had passed, Luke felt that he had repented, and spilled his food on the floor. He went to his cubical and resumed his life as usual. His elders became worried, but they decided to say nothing.

Note. The primary causal pivot is underlined in the standard story, as are its corresponding statements in each of the variants.

shuffled). Each story pair was presented on a separate page, with the top story always labeled "Story $A$ " and the bottom one "Story B".

Design. Pair type was counterbalanced within subjects, so that each subject received an equal number of deletion, replacement, and shuffled pairs. Order of standard-variant presentation within each pair was counterbalanced both within and between subjects. Story pair assignment within each story set was also counterbalanced, and order of story pair presentation was randomized.

Procedure. Subjects were instructed that for each story pair, they should indicate which direction of comparison they preferred. Subjects responded by completing the following statement with the appropriate letters (A and B): "Story __ is more similar to Story _ than the reverse."

\section{Results and Discussion}

As predicted, there was an overall asymmetry in similarity favoring the variant-to-standard direction. The proportion of responses in this direction $(m=.69)$ was significantly greater than chance $(\mathrm{z}=11.46 ; \mathrm{p}<.001)$. Further, this asymmetry was observed for each variant condition, and the proportions of responses in the predicted direction for DV $(m=.61)$, RV $(m=.73)$, and $S V(m=.73)$ pairs were all significantly greater than chance $(z=3.97, p<.001 ; z=7.96, p<.001 ;$ and $z=7.93$, $\mathrm{p}<.001$, respectively).

Our second prediction concerning the relationship between degree of coherence imbalance and degree of asymmetry was also borne out. An analysis of variance performed across items on the proportion of responses in the predicted direction showed a significant main effect for Variant Condition $(F(2,34)=8.89, p<.001)$. Planned comparisons indicated that the asymmetries for RV and SV pairs were significantly greater than the asymmetry for DV pairs $(t(17)=3.65, \mathrm{p}<.005$; and $\mathrm{t}(17)=3.59, \mathrm{p}<.005$, respectively). Across all items there was a positive correlation between the pretest coherence imbalance ratings and the proportion of responses in the predicted direction $(r(52)=.53, p<.001)$.

One slightly odd finding was that the asymmetries for the RV and SV pairs did not differ, despite the greater coherence imbalance rating for the SV condition. In keeping with the structural alignment approach, we hypothesized that this could be due to a difference in alignability. It is likely that the SV pairs were significantly less alignable than either the deletion or replacement pairs, as the variants in these pairs exhibited less causal structure. Because coherence imbalance is calculated over aligned structures, alignability is necessary for directionality. Thus, if the alignability of the shuffled pairs was significantly lower than that for the other narrative pairs, the potential for exhibiting directionality would be lower. To test this possibility, we conducted a stimulus posttest, in which subjects were presented with the above story pairs and asked to rate their alignability on a scale of 1 (low alignability) to 7 (high alignability). As predicted, significantly lower alignability ratings were obtained for the $S V$ pairs $(m=4.09)$ than for the DV pairs $(m=5.29)$ and the $R V$ pairs $(m=5.09)(t(17)=-7.00, p<.001$, and $t(17)=-6.40$, p<.001, respectively).

\section{Experiment 2}

Our results so far are consistent with the claim that there is a functional basis to asymmetries in similarity: people prefer the more coherent item in the base position of a comparison. However, one crucial implication of our position has not yet been addressed: namely, the claim that 
the coherence imbalance preference arises because the direction of a structural alignment influences the potential inferential carryover (and thus, the degree of potential informativity). In Experiment 2, we tested the relationship between asymmetry and informativity by asking subjects to produce inferences between the paired narratives used in Experiment 1. We predicted that more inferences would be generated from standard to variant stories than in the reverse direction.

However, we would not necessarily expect the degree of inference directionality to reflect the degree of coherence imbalance across all three variant conditions. Specifically, inference generation should be easiest in the DV and RV story pairs. This is because the variants in each of these conditions contain a definable gap or anomaly in the causal structure that can serve to direct predicate attributions. In contrast, the variants in the SV story pairs display a general lack of structure with no clearly aligned gaps for inferences. Further, although these variants may lack some implied causality inferable from sequencing, they contain the same basic propositions as their corresponding standards. Thus, the degree of inference directionality should be significantly lower for these story pairs. This prediction is underscored by the finding discussed above that the SV pairs were rated as less alignable than either of the other two types of story pairs. Because inference depends on structural alignment (Clement \& Gentner, 1991), the lower alignability of these story pairs should impede predication.

\section{Method}

Subjects. Forty-eight Northwestern University undergraduates participated in partial fulfillment of a course requirement. None of these had participated in Experiment 1.

Materials and Design. The materials and design were identical to those used in Experiment 1.

Procedure. Subjects were instructed that for each story pair, they should make an inference concerning one of the stories based on the content of the other. They were informed that an inference could be "anything at all that you decide might be true of the story". Subjects were asked to (1) indicate which story they were making their inference about; and (2) write out their inference in the space provided.

\section{Results and Discussion}

As predicted, there was an overall asymmetry in inference generation favoring the standard-to-variant direction. The proportion of responses in this direction $(m=.80)$ was significantly greater than chance $(z=17.73, p<.001)$. Further, this asymmetry was observed for each variant condition, and the proportions of responses in the predicted direction for $\mathrm{DV}(\mathrm{m}=.85), \mathrm{RV}(\mathrm{m}=.82)$, and $\mathrm{SV}(\mathrm{m}=.73)$ pairs were all significantly greater than chance $(\mathrm{z}=11.86$, $p<.001 ; \quad z=10.96, p<.001 ; \quad$ and $z=7.88, p<.001$, respectively).

Our second prediction, that the degree of inferential asymmetry would vary with differences in the structural alignment of the story pairs, was also borne out. An analysis of variance performed across items on the proportion of responses in the predicted direction showed a significant main effect for Variant Condition $(F(2$,
$34)=13.52, p<001)$. Planned comparisons indicated that the inferential asymmetry for the $S V$ pairs was significantly less than that for the DV and RV pairs $(t(17)=-4.51, p<.001$; and $t(17)=-3.85, p<.001$, respectively). Further, across all items there was a small but significant positive correlation between the alignability ratings obtained above and the inference asymmetry ratings $(r(52)=.32, p<.05)$, supporting the position that alignability is necessary for inference generation.

\section{Experiment 3}

In Experiment 2, we found that the degree of inferential asymmetry favoring the more coherent item as the base depended on the structural alignment of the story pairs. Although this finding was predicted, it raises a question about how all of these predictions fit together. We have argued that the preferred direction of comparison is governed by coherence imbalance, and that this direction maximizes the degree of perceived informativity of a comparison. But in this case, why did the SV pairs, which exhibited the lowest inferential asymmetry in Experiment 2, show the highest degree of directional asymmetry in Experiment 1 ? The answer to this question lies in a deeper consideration of the means by which comparisons may be informative. Although inference generation is a most potent means of providing novel information in a comparison, there are at least two other structurally derived types of informativity: conceptual restructuring and structural highlighting. In conceptual restructuring, the elements of the common relational system are equally present in both comparison referents, but exhibit less mutual constraint in the target than in the base. In this type of situation, the comparison does not so much involve attributions from the base to the target as it does restructuring the target objects in terms of their relational roles in the base. In contrast, structural highlighting involves comparisons in which either (1) the common relational system is equally present in both comparison referents, but less prominent in the target; or (2) multiple possible structures are being entertained for the objects in the target, only one of which is alignable with the base. Structural highlighting leads to greater attentional focus on the common structure in the target. Although it may seem trivial compared to inference generation and conceptual restructuring, we believe that promoting focus on a particular system may at times be important in learning (see Gentner, Ratterman, Markman, \& Kotovsky, in press; Gick \& Holyoak, 1983).

Although the SV condition was found to exhibit a lower degree of inference directionality, these comparisons are obvious candidates for conceptual restructuring or structural highlighting. In Experiment 3, we asked subjects to directly rate the informativity of forward and reverse comparisons between stories drawn from the DV and SV pairs used in Experiments 1 and 2 . In keeping with the functional approach, we predicted that the forward direction of comparison (variant to standard) would be rated as more informative than the reverse direction (standard to variant), and that the difference in informativity between these two directions would be greater for the SV pairs than the DV 
pairs. In other words, although the SV pairs displayed a lower degree of inferential asymmetry than the DV pairs, their degree of asymmetry in rated informativity should be higher than that of the DV pairs, reflecting the additional potential for restructuring and highlighting. If this result is obtained, it will (we suggest) explain the high directional asymmetry of the SV pairs obtained in Experiment 1.

\section{Method}

Subjects. Thirty-two Northwestern University undergraduates participated in partial fulfillment of a course requirement. None of these had participated in Experiments 1 or 2.

Materials. The stimuli were pairs of stories drawn from 12 of the 18 basic story sets used in Experiments 1 and 2. Each pair consisted of a standard and one of two variants (deletion or shuffled). Each story pair was presented on a separate page, with the top story always labeled "Story $A$ " and the bottom one "Story B".

Design. Pair type was counterbalanced within subjects, so that each subject received an equal number of deletion and shuffled pairs. Order of standard-variant presentation within each pair was counterbalanced both within and between subjects. Story pair assignment within each story set was also counterbalanced, and order of story pair presentation was randomized.

Procedure. Subjects were instructed that for each story pair, they should rate the degree of informativity of the comparison of Story A to Story B on a scale of 1 (very uninformative) to 7 (very informative).

\section{Results and Discussion}

An analysis of variance performed across items on the rated informativity of the comparisons found a significant main effect for Direction, such that forward comparisons $(m=5.15)$ were rated as more informative than reverse comparisons $(m=2.45)(F(1,11)=185.54, p<.001)$. Further, the predicted interaction between Direction and Variant Condition was also obtained, such that the difference in informativity between the two directions was significantly greater for the SV pairs $(m=3.39)$ than for the DV pairs $(m=2.01)$ $(F(1,11)=7.90, p<.025)$. Thus, the differential perceived informativity of the comparisons is predicted by their degree of coherence imbalance, even when candidate inferences are not possible. It appears that the functional value of this coherence imbalance preference results from the potential for restructuring and highlighting, as well as from the potential for generating new inferences.

\section{Summary}

Research on asymmetry in similarity, metaphor, and analogy has often focused on different sources of directionality for each of these comparison types (e.g., Ortony, 1979; Glucksberg \& Keysar, 1990). In contrast, we have suggested that a major source of asymmetry in comparisons of all types is coherence imbalance. Further, we have argued that this approach has the advantage of providing a functional perspective to directional comparison: it posits that asymmetries reflect the perceived informativity of comparisons. Unlike feature-based approaches, the structure-mapping account provides a clear mechanism for inference generation, as well as for conceptual restructuring and structural highlighting.

In formulating the coherence imbalance hypothesis, we have operationalized coherence as systematicity, or the degree to which a concept is structured by a system of predicates govemed by higher-order causal or explanatory relations. This notion is clearly related to text-processing approaches to coherence, especially those that emphasize the causal connectivity of situation models (e.g., Kintsch \& van Dijk, 1978; Trabasso \& van den Broek, 1985). However, this definition of coherence is also in keeping with recent theorizing on the structure of everyday concepts such as horse or hammer (e.g., Keil, 1989; Murphy \& Medin, 1985), in which coherence is derived both internally (from causal or functional correlations between attributes) and externally (from the position of the concept in causal or explanatory networks existing between multiple concepts). Thus, although our experiments involved comparisons between narrative structures, the basic findings are clearly applicable to comparisons between entities denoted by single lexical units.

For example, we began our discussion of asymmetry with the example of a comparison between North Korea and Red China, for which a clear directional preference exists. According to the present account, the reason that the comparison of North Korea to Red China is preferred over the reverse is that the representation of China possesses a greater degree of systematicity: our knowledge of the country (e.g., its history, culture, economy, and relations with other countries) is more highly structured than that of North Korea. This allows for the comparison to be informative, and enables us to make candidate inferences about North Korea such as how communism interacts with traditional culture, how student dissident are treated, and so on.

The coherence imbalance hypothesis also suggests an explanation for different asymmetric trends across different comparison types. For example, metaphors tend to display greater directional asymmetry than similarity comparisons (Ortony, Vondruska, Foss, \& Jones, 1985). On the present account, this would suggest that metaphors should exhibit greater coherence imbalance than literal comparisons. This suggestion is supported by Connor and Kogan (1980), who note that metaphoric targets tend to be ambiguous, possessing unknown or uncertain properties. Thus, the informativity potential is greater for metaphors than literal comparisons. Of course, this account is in may ways compatible with Glucksberg and Keysars's (1990) classinclusion model of metaphor, in which the concept that is best able to provide a predicating category is the preferred metaphoric base. However, the scope of their model is limited to metaphoric statements, whereas the structural alignment account that we are advocating applies generally across comparison types. Further, unlike the structural alignment account, the class-inclusion model provides no clear process for inference selection (Wolff \& Gentner, 1992, in preparation).

In this paper, we have focused our attention on structural determinants of asymmetry, as they provide the most 
obvious mechanisms for lending structure to comparison targets. Of course, coherence imbalance is context dependent, as the relational structure of a concept placed in alignment depends not only on the representation to which it is being compared, but also on the purpose of the comparison. Further, we would not wish to claim that coherence imbalance is the only source of asymmetry in comparisons. For example, the comparison snow is like salt may be preferred over salt is like snow. In this case, the asymmetry seems to stem from a salience imbalance of certain shared features of the target and base (e.g., whiteness and granularity), rather than from more structural considerations. However, we suggest that such attributebased comparisons are less frequent in normal discourse than the richer and more informative comparisons (such as that between North Korea and Red China) that we have been discussing. The coherence imbalance hypothesis takes a structural alignment approach to the process of comparison, from which emerges an account of asymmetries that is inherently functional.

\section{Acknowledgements}

This work was supported by NSF grant BNS 87-20301 and ONR grant N00014-89-J1272 awarded to the first author.

\section{References}

Clement, C., \& Gentner, D. (1991). Systematicity as a selection constraint in analogical mapping. Cognitive Science, 15, 89-132.

Connor, K., \& Kogan, N. (1980). Topic-vehicle relations in metaphor: The issue of asymmetry. In R. P. Honeck \& R. R. Hoffman (Eds.), Cognition and figurative language. Hillsdale, NJ: LEA.

Falkenhainer, B., Forbus, K. D., \& Gentner, D. (1989). The structure-mapping engine: Algorithm and examples. Artificial Intelligence, 41, 1-63.

Gentner, D. (1983). Structure-mapping: A theoretical framework for analogy. Cognitive Science, 7, 155-170.

Gentner, D. (1989). The mechanisms of analogical learning. In S. Vosniadou \& A. Ottony (Eds.), Similarity and analogical reasoning. Cambridge: Cambridge University.

Gentner, D., \& Clement, C. (1988). Evidence for relational selectivity in interpreting analogy and metaphor. In G. Bower (Ed.), The psychology of learning and motivation. New York: Academic Press.

Gentner, D., \& Markman, A. B. (1993). Analogy - watershed or waterloo? Structural alignment and the development of connectionist models of analogy. In S. J. Hanson, J. D. Cowan, \& C. L. Giles (Eds.), Advances in neural information processing systems. San Mateo, CA: Morgan Kaufmann.

Gentner, D., et al (in press). Two forces in the development of relational similarity. To appear in G. Halford \& T. Simon (Eds.), Developing cognitive competence: New approaches to process modeling. Hillsdale, NJ: LEA.

Gick, M. L., \& Holyoak, K. J. (1983). Schema induction and analogical transfer. Cognitive Psychology, 15, 1-38.
Glucksberg, S., \& Keysar, B. (1990). Understanding metaphorical comparisons: Beyond similarity. Psychological Review, 97, 3-18.

Keil, F. C. (1989). Concepts, kinds, and cognitive development. Cambridge: MT.

Kintsch, W., \& van Dijk, T. A. (1978). Towards a model of text comprehension and production. Psychological Review, 85 , 363-394.

Markman, A. B., \& Gentner, D. (1990). Analogical mapping during similarity judgments. Proceedings of the Twelfth Annual Conference of the Cognitive Science Society. Hillsdale, NJ: LEA.

Markman, A. B., \& Gentner, D. (1993). Splitting the differences: A structural alignment view of similarity. Journal of Memory and Language, 32, 517-535.

Medin, D. L., Goldstone, R. L., \& Gentner, D. (1993). Respects for similarity. Psychological Review, 100, 254-278.

Murphy, G. L., \& Medin, D. L. (1985). The role of theories in conceptual coherence. Psychological Review, 92, 289. 316.

Ortony, A. (1979). Beyond literal similarity. Psychological Review, 86, 161-180.

Ortony, A., et al (1985). Salience, similes, and the asymmetry of similarity. Journal of Memory and Language, 24, 569. 594.

Tourangeau, R., \& Rips, L. (1991). Interpreting and evaluating metaphors. Journal of Memory and Language, 30, 452-472.

Trabasso, T., \& van den Broek, P. (1985). Causal thinking and the representation of narrative events. Journal of Memory and Language, 24, 612-630.

Tversky, A. (1977). Features of similarity. Psychological Review, 84, 327-352.

Wolff, P., \& Gentner, D. (1992). The time course of metaphor comprehension. Proceedings of the Fourteenth Annual Conference of the Cognitive Science Society. Hillsdale, NJ: LEA.

Wolff, P., \& Gentner, D. (in preparation). Metaphor comprehension: Comparison or class inclusion? 